\title{
Esencialismo psicológico y justificación del sistema en la percepción del cambio social
}

\section{Psychological Essentialism and System Justification in the face of Social Change Essencialismo psicológico e justificação do sistema na percepção da mudança social}

\author{
Luisa Ramírez, Diana Carolina Camargo, Valentina Charry, \\ María Fernanda Osorio, Angie Ramírez, María Sighinolfi* \\ Universidad del Rosario
}

Doi: dx.doi.org/10.12804/apl33.01.2015.11

\section{Resumen}

Evidencia empírica reciente sugiere que las creencias esencialistas sobre los grupos sociales suelen estar relacionadas con actitudes de prejuicio y discriminación hacia estos. Esta relación es moderada por aspectos como el estatus del perceptor social relativo al grupo evaluado y por las creencias sobre la estabilidad del sistema social. Esta investigación se propuso evaluar, en primer lugar, la estructura de las creencias sobre la orientación sexual. En segundo, la relación entre la creencia en la estabilidad del sistema y el uso de argumentos esencialistas en un grupo normativo (heterosexuales) y un grupo no normativo (homosexuales) en una muestra de comunidad colombiana. Para lograrlo, se creó un diseño multifactorial de 3 (manipulación experimental: percepción de cambio inminente, percepción de estabilidad y grupo control) x 2 (orientación sexual: homosexual vs. Heterosexual), introduciendo esencialismo como variable dependiente. Los resultados sugieren que las creencias sobre la orientación sexual se agrupan alrededor de dos dimensiones esencialistas aquí denominadas inmutabilidad y fundamentalidad. Adicionalmente, sugieren que las creencias esencialistas en la inmutabilidad predicen actitudes más favorables hacia las personas homosexuales que las creencias esencialistas en la fundamentalidad de la orientación sexual. Las implicaciones de estos hallazgos son discutidas.

Palabras clave: Esencialismo, orientación sexual, teorías legas, prejuicio.

* Luisa Ramírez, Programa de Psicología, Universidad del Rosario; Diana Carolina Camargo, Programa de Psicología, Universidad del Rosario; Valentina Charry, Programa de Psicología, Universidad del Rosario; María Fernanda Osorio, Programa de Psicología, Universidad del Rosario; Angie Ramírez, Programa de Psicología, Universidad del Rosario; María Sighinolfi, Programa de Psicología, Universidad del Rosario.

Este proyecto fue co-financiado por la Universidad del Rosario y el Departamento Administrativo de Ciencia, Tecnología e Innovación, Colciencias, como parte del programa de jóvenes investigadores e innovadores, Convenio Especial de Cooperación 0064/2012, en el contexto del semillero de Investigación en Cognición Social.

La correspondencia relacionada con este artículo debe ser dirigida a Luisa Ramírez, Programa de Psicología, Universidad del Rosario, Bogotá, Carrera 24 \# 63C-69. Correo electrónico: luisa.ramirez@urosario.edu.co.

Para citar este artículo: Ramírez, L., Camargo, D. C., Charry, V., Osorio, M. F., Ramírez, A., \& Sighinolfi, M. (2015). Esencialismo psicológico y justificación del sistema en la percepción del cambio social. Avances en Psicología Latinoamericana, 33(1), 157-174. doi: dx.doi.org/10.12804/ap133.01.2015.11 


\section{fibstract}

Recent empirical evidence suggests that essentialist beliefs about social groups are often associated with prejudice and discrimination against them. This relation is moderated by social status and beliefs about system stability. This study aimed at: first, evaluating the factorial structure of beliefs about sexual orientation. Second, evaluating the effect of beliefs in system stability and the use of essentialist arguments within a normative social category (heterosexuals) and a non-normative one (homosexuals) in a community sample in Colombia. To test this relationship we created a multifactorial design of 3 (belief in system stability: imminent change, stability and control) x 2 (sexual orientation), introducing essentialist beliefs about sexual orientation as a dependent variable. Findings suggest that beliefs about sexual orientation load on two essentialist dimensions: immutability and fundamentality. Also, findings suggest that beliefs in the immutability of sexual orientation are unrelated, whereas the beliefs about the fundamentality of sexual orientation significantly relate to prejudice and discrimination among heterosexual but not among homosexual people. The implications of these findings are discussed.

Keywords: Essentialism, sexual orientation, lay theories, prejudice.

\section{Resumo}

Evidência empírica recente sugere que as crenças essencialistas sobre os grupos sociais costumam estar relacionadas com atitudes de prejuízo e discriminação aos mesmos. Esta relação és moderada por aspectos como o status do preceptor social relativo ao grupo avaliado, e pelas crenças sobre a estabilidade do sistema social. Esta pesquisa se propôs avaliar em primeiro lugar, a estrutura das crenças sobre a orientação sexual. Em segundo lugar, a relação entre a crença na estabilidade do sistema e o uso de argumentos essencialistas em um grupo normativo (heterossexuais) e um grupo não normativo (homossexuais) uma amostra de comunidade colombiana.
Para consegui-lo criamos um desenho multifatorial de 3 (manipulação experimental: percepção de mudança iminente, percepção de estabilidade e grupo controle) x 2 (orientação sexual: homossexual vs. heterossexual), introduzindo essencialismo como variável dependente. Os resultados sugerem que as crenças sobre a orientação sexual se agrupam em torno de duas dimensões essencialistas aqui denominadas imutabilidade e fundamentalidade. Adicionalmente, sugerem que as crenças essencialistas na imutabilidade predizem atitudes mais favoráveis às pessoas homossexuais, que as crenças essencialistas na fundamentalidade da orientação sexual. As implicações destes achados são discutidas.

Palavras-chave: Essencialismo, orientação sexual, teorías legas, prejuízo

Para predecir y ganar certeza sobre el mundo que les rodea, los seres humanos adoptan teorías de sentido común o teorías legas, que explican la personalidad, la naturaleza humana, la naturaleza de los grupos sociales y muchos otros aspectos de la vida social (Estrada, Oyarzún \& Yzerbyt, 2007; Heider, 1958). Una teoría lega que explica la naturaleza de los grupos sociales es el esencialismo psicológico, el cual se caracteriza por la creencia en que una esencia común y profunda genera las propiedades superficiales de algunos grupos sociales (Medin \& Ortony, 1989). El pensamiento esencialista se desarrolla desde edades tempranas (Gelman, 2003; Hirschfeld, 2002), entre personas de diversas etnias y culturas (Mahalingam \& Rodríguez, 2003; Morton, Hornsey \& Postmes, 2009; Verkuyten, 2003), nacionalidades (Pereira, Álvaro \& Schweiger, 2010; Tsukamoto, Enright \& Karasawa, 2013) y estatus sociales (Mahalingam \& Rodríguez, 2006; Verkuyten, 2003).

Varias investigaciones han intentado identificar la estructura subyacente a las creencias sobre la orientación sexual (Arseneau, Grzanka, Miles \& Fassinger, 2013; Haslam \& Levi, 2006; Hegarty \& Pratto, 2001). Hegarty y Pratto (2001), por ejemplo, encontraron que existen creencias en las que se 
afirma que la orientación sexual se determina tempranamente durante el ciclo vital (determinación temprana), que es un principio básico organizador de la identidad (identidad esencial), inmutable una vez emerge (estabilidad en la adultez) y que las personas pueden ser divididas en dos categorías, basadas en su orientación sexual (binario heterosexualidad/homosexualidad), las cuales se agrupan en dos dimensiones: inmutabilidad (creencia en que la orientación sexual está determinada por la biología, durante la infancia temprana y es estable a lo largo de la vida) y fundamentalidad (creencia en que la orientación sexual es parte central de la identidad y en que existen diferencias fundamentales entre homosexuales y heterosexuales).

En el mismo sentido, Haslam y Levi (2006) encontraron que las creencias sobre la gente gay se agrupan en dos dimensiones que denominaron clases naturales (que incluye la creencia en la naturalidad, inmutabilidad y determinación temprana de la categoría) y entitatividad (que agrupa las creencias en una estructura binaria y características necesarias). Posteriormente, encontraron evidencia de una tercera dimensión a la que llamaron universalidad, para referirse a la estabilidad histórica y cultural de la homosexualidad. Más recientemente, Arseneau, Grzanka, Miles y Fassinger (2013) encontraron cuatro dimensiones: (a) naturalidad (agrupando creencias en la determinación temprana, la naturalidad, la inmutabilidad y la invariabilidad cultural), (b) discreción (estructura binaria y exclusividad), (c) homogeneidad (uniformidad de las personas con una misma orientación sexual), (d) informatividad (potencial inductivo de la categoría).

Dado esto, las creencias en la naturalidad, la inmutabilidad y la determinación temprana de la orientación sexual aparecen fuertemente asociadas. En contraste, si bien las creencias en la homogeneidad, informatividad, fundamentalidad, exclusividad y discreción de la categoría forman parte de la estructura que organiza las creencias sobre la orientación sexual, su asociación es menos estable. Quizá sea por esta razón que la mayor parte de las investigaciones han enfatizado la dimensión de clases naturales y, en particular, las creencias en la determinación biológica (atribución de las diferencias entre grupos sociales a causas de orden biológico, genético o natural) y la inmutabilidad (estabilidad) de la categoría (Dar-Nimrod \& Heine, 2011; Haider-Markel \& Joslyn, 2008; Jayaratne et al., 2006; Keller, 2005). Estos estudios han encontrado relaciones estadísticamente significativas entre estas creencias y actitudes positivas hacia la gente homosexual.

No obstante, las creencias esencialistas se pueden organizar de diversas maneras. Rangel y Keller (2011), por ejemplo, encontraron evidencia de la existencia de creencias en el determinismo social (las características superficiales de los grupos sociales están determinadas por factores sociales como la crianza temprana, las condiciones socioeconómicas, etc.) en el pensamiento lego sobre los grupos sociales y encontraron que en Alemania esta creencia tiene una capacidad predictiva independiente de la creencia en el determinismo genético. Consistentemente, señalaron que no solo las creencias en el determinismo biológico (genes), sino también social (condiciones de crianza), e incluso metafísico (Dios) podrían constituir argumentos esencialistas en algunos contextos.

De este modo, aparentemente las creencias esencialistas sobre los grupos sociales, y en particular sobre la población gay, varían en su organización (alrededor de varias dimensiones) y contenido (argumentos de determinismo biológico, social e incluso metafísico) en las diferentes poblaciones en que han sido evaluadas. Esto podría indicar que el pensamiento esencialista es flexible y se adapta a diferentes contextos, con diferentes implicaciones para los grupos socialmente relevantes en cada caso (Jayaratne et al. 2009; Mahalingham, 2003; Verkuyten, 2003). 


\section{Creencias esencialistas sobre la gente homosexual en Colombia}

En un estudio cualitativo realizado en Bogotá con hombres y mujeres homosexuales, Estrada, Acuña, Camino y Traverso (2007) encontraron que los participantes de orientación sexual gay concebían su orientación sexual como algo cambiante y plástico, producto de las experiencias personales (argumentos opuestos al esencialismo). En otras ocasiones, estas personas consideraron que la homosexualidad estaba determinada por factores como las relaciones familiares deficientes o las experiencias de abuso (creencia en la determinación temprana o determinismo social). No obstante, una vez definida la orientación sexual, esta era percibida como algo inmutable (Estrada, et al., 2007). En otro estudio cualitativo, Ramírez y Moreno (2013) compararon las creencias de hombres homosexuales y heterosexuales y encontraron que entre participantes heterosexuales la orientación sexual era entendida como una enfermedad o como el resultado de un daño por el cual algunos hombres, desde que nacen piensan y sienten como mujeres. Así mismo, encontraron creencias en las que la orientación sexual es el resultado de experiencias tempranas de crianza relacionadas con el aprendizaje del rol de género y el modelamiento (ej. los hombres no lloran) o bien, de experiencias tempranas traumáticas (ej. abuso). Para algunos hombres heterosexuales, los comportamientos afeminados permiten distinguir entre homosexuales y heterosexuales y están presentes desde el nacimiento. No obstante, los participantes parecen concebir la masculinidad como algo mutable. Por otra parte, entre hombres gais también apareció evidencia de la creencia en la determinación temprana de la orientación sexual, fundamentada en determinantes sociales como las experiencias tempranas de crianza y experiencias sexuales traumáticas, pero no en determinantes biológicos. Entre estos últimos, la orientación sexual (una vez establecida) apareció como algo inmutable.
Los hallazgos de las dos investigaciones realizadas en Bogotá indican que tanto homosexuales como heterosexuales recurren, en algunas ocasiones, a argumentos esencialistas pero no necesariamente de determinismo biológico.

\section{Esencialismo psicológico y prejuicio social}

Con frecuencia, se ha encontrado que la creencia en la determinación genética y la inmutabilidad de la orientación sexual se relacionan con menores niveles de prejuicio en varias culturas (Jayaratne et al., 2006; Keller, 2005; Ramírez, 2007). Para algunos, la relación positiva entre el prejuicio y la creencia en la naturalidad de la homosexualidad se debe a que la creencia en una base biológica remueve las atribuciones de control sobre la orientación sexual, lo cual reduce la culpa y la responsabilidad moral resultante si la homosexualidad es entendida como una decisión personal (Dar-Nimrod \& Heine, 2011; Haider-Markel \& Joslyn, 2008; Jayaratne et al., 2006).

Otros estudios, sin embargo, han encontrado que diferentes dimensiones del pensamiento esencialista se relacionan positiva y negativamente con el prejuicio hacia este grupo social (Haslam, et al., 2002; Hegarty \& Pratto, 2001). Por ejemplo, las creencias en la entitatividad, en la discreción y en el potencial inductivo de la homosexualidad han estado asociadas con actitudes negativas, mientras las creencias en la inmutabilidad, la naturalidad y la estabilidad se han asociado con actitudes positivas hacia lesbianas y gais (Haslam \& Levy, 2006; Haslam et al., 2000; Hegarty \& Pratto, 2001). Esta variabilidad en el uso de los argumentos esencialistas hacia los grupos sociales ha llevado a algunos autores a plantear la posibilidad de que, si bien es posible que el pensamiento esencialista conlleve algunas implicaciones favorables o desfavorables para determinados grupos sociales, también es posible que las personas utilicen estos argumentos a manera de ideologías legitimadoras para defender o 
justificar el estatu quo. De hecho, existe evidencia que apunta a favor de esta noción, al señalar que grupos de diferente estatus social (normativo o no normativo) utilizan el esencialismo de diferentes maneras y con diferentes implicaciones para los grupos sociales evaluados (Dar-Nimrod \& Heine, 2011; Mahalingam \& Rodríguez, 2003; Morton, Hornsey \& Postmes, 2009; Verkuyten, 2003).

\section{Implicaciones del esencialismo psicológico sobre las relaciones sociales}

La teoría de Justificación del Sistema afirma que las personas se encuentran motivadas para defender el orden social predominante, lo cual permite mantener la creencia en que viven en un mundo predecible y seguro (Blasi \& Jost, 2006). La mayor parte de las investigaciones en el tema se han enfocado en la situación de grupos étnicos/raciales, de género $\mathrm{y}$, en menor medida, en otros como los grupos políticos, económicos y académicos (Jost \& Pelham, 2003). La presencia de justificación del sistema entre grupos con orientaciones sexuales diversas no ha sido objeto de mucha investigación hasta donde se sabe, salvo contadas excepciones (Jost et al. 2003). No obstante, es posible que el pensamiento esencialista sobre la orientación sexual tenga consecuencias importantes, debido a que permite racionalizar las diferencias existentes entre grupos homosexuales y heterosexuales, al atribuir las desigualdades a aspectos inherentes al grupo social y no a los arreglos sociales dominantes. De ahí que algunos investigadores hayan argumentado que las creencias esencialistas constituyen una ideología justificadora del estatu quo (Keller, 2005; Verkuyten, 2003; Yzerbyt, Rocher \& Schadron, 1997). Hasta donde se tiene conocimiento, la noción de que el esencialismo psicológico sirve como ideología justificadora del estatu quo en relación con los grupos de orientación sexual diversa no ha sido evaluada aún.

Consecuentemente, esta investigación se propuso evaluar el uso y las implicaciones del pensamiento esencialista en el grupo normativo (heterosexuales) y en el grupo no normativo (homosexuales) en dos situaciones: cuando existe la percepción de (a) estabilidad o (b) cambio en el sistema vigente de relaciones. En la última década en Colombia, los debates sociales y políticos en torno al acceso a derechos de manera igualitaria para la población homosexual han ganado visibilidad y se han convertido en un punto de gran relevancia en la agenda política y progresivamente se han venido reconociendo los derechos a este grupo de población. Recientemente, las discusiones en torno al matrimonio igualitario, la definición de familia y el derecho a la adopción por parejas homosexuales han estado sobre la mesa. A inicios de 2013, una sentencia de la Corte Constitucional (C-577 12 de julio de 2011) generó un gran debate, pues establecía que si para el 20 de junio del mismo año el Congreso no había legislado sobre el matrimonio igualitario en Colombia, este se empezaría a aplicar automáticamente. El 30 de julio de 2014 el Consejo de Estado reconoció el derecho de cualquier persona, sin importar su orientación sexual, a establecer una familia, y el 28 de agosto de 2014 la Corte Constitucional aprobó por primera vez la adopción conjunta de una niña por sus madres. Así entonces, la realización de este experimento que inició en el primer semestre de 2013 tuvo lugar en un periodo en el que la expectativa de un cambio histórico en el sistema de relaciones entre heterosexuales y homosexuales era creíble.

\section{Método}

Para evaluar la estructura de las creencias sobre la orientación sexual y el efecto de la percepción de estabilidad o cambio social sobre el uso de argumentos esencialistas entre población normativa y no normativa, se llevó a cabo primero un análisis factorial de las creencias esencialistas, seguido por un diseño multifactorial de 3 (manipulación experimental: percepción de cambio inminente, percepción de estabilidad y grupo control) x 2 
(orientación sexual: homosexual y heterosexual), y se introdujo el esencialismo como variable dependiente. Posteriormente, se realizó un análisis de regresión para evaluar la relación entre creencias esencialistas y el posible papel moderador de la percepción de estabilidad o cambio en el sistema sobre actitudes de prejuicio y discriminación.

\section{Participantes}

Se tomó una muestra de 233 sujetos, a los que se les aplicó un cuestionario. Los 233 cuestionarios respondidos por los sujetos fueron asignados de manera aleatoria a una de las dos condiciones experimentales (percepción de cambio inminente $(\mathrm{n}=47)$ o percepción de estabilidad $(\mathrm{n}=46)$, o al grupo control $(\mathrm{n}=115)$. El rango de edad de los participantes variaba entre 18 y 70 años $(\mathrm{M}=$ 31.7, DS =12.3). La técnica de muestreo fue por conveniencia. Se eliminaron cuestionarios por presentar patrones de respuesta sospechoso, no comprender la lectura de la manipulación o información faltante, quedaron 209 cuestionarios (103 de hombres, 103 de mujeres y 3 de personas que no reportaron su sexo).

\section{Procedimiento}

Inicialmente, se realizó un pilotaje de todos los instrumentos para observar que sus propiedades psicométricas fueran adecuadas. Los participantes en las dos condiciones experimentales, una vez efectuado el primer contacto, recibieron un cuestionario que contenía un formato de consentimiento informado, la manipulación experimental y las escalas de medición que incluían esencialismo, conservadurismo, prejuicio y los ítems para evaluar aprobación del matrimonio igualitario y la adopción por parejas homosexuales. La mayoría de participantes en las condiciones experimentales contestaron la encuesta en cuarenta minutos aproximadamente, los del grupo control tomaron en promedio veinticinco minutos para responderla.
Los participantes en las condiciones experimentales leyeron una de las dos manipulaciones diseñadas para inducir la percepción de un cambio inminente o de estabilidad; respondieron el chequeo de la manipulación para luego diligenciar las escalas de esencialismo psicológico, prejuicio, actitudes hacia el matrimonio y la adopción y datos sociodemográficos (el orden de las escalas se aleatorizó). Los participantes en el grupo control no recibieron manipulación.

\section{Instrumentos}

Manipulación experimental. La manipulación consistió en la creación de dos versiones de un artículo de periódico ficticio, con el fin de activar en los participantes la percepción de cambio o de estabilidad del sistema. Cada artículo tenía siete párrafos de longitud y aproximadamente 615 palabras. La versión de cambio social enfatizó los logros de la gente gay en materia de aceptación social y derechos civiles. La versión de estabilidad enfatizó la falta de garantías para el cumplimiento de los derechos civiles que ha adquirido este grupo (ver Anexo).

Chequeo de la manipulación. Para establecer la efectividad de la manipulación se les solicitó a los participantes en las condiciones experimentales identificar la idea central del artículo (cambio inminente o estabilidad) que habían leído. Se construyó una variable dicótoma para registrar esta información $(0=$ no identifica la idea principal; $1=$ identifica la idea principal). Los participantes que no respondieron adecuadamente a esta pregunta $(n$ $=7$ ) se excluyeron del análisis.

\section{Creencias esencialistas sobre la orientación se-} xual. Los ítems de las escalas de Haslam y Levy (2006) y Hegarty y Pratto (2006) fueron adaptados y traducidos para construir una escala de esencialismo que indagara por las creencias en la naturalidad, inmutabilidad, informatividad, discreción, 
determinación temprana y la estabilidad cultural e histórica de la categoría $(\alpha=, 68)$.

Actitudes hacia hombres y mujeres homosexuales. Para la medición de prejuicio se utilizaron dos versiones de cinco ítems de la escala de actitudes hacia hombres gais (ATG Scale) y lesbianas (ATL Scale) tomadas de Herek (1998). Estas escalas han mostrado fuertes propiedades psicométricas en el pasado. En este caso, la confiabilidad fue bastante alta $(\alpha=.82$ y $\alpha=.84$ respectivamente).

Variables demográficas. Esta sección del cuestionario indagó por la edad, sexo, religión, nivel educativo, estatus socio-económico y orientación sexual (tabla 1).

Tabla 1

Descripción de las características demográficas de los grupos de participantes

\begin{tabular}{llc}
\hline & Variables Sociodemográficas & $n$ \\
\hline \multirow{2}{*}{ Sexo } & Hombre & 109 \\
& Mujer & 102 \\
Edad & $18-40$ & 161 \\
& $41-79$ & 50 \\
Nivel & Tecnológico o inferior & 82 \\
Educativo & Universitario o superior & 128 \\
& & \\
$\begin{array}{l}\text { Orientación } \\
\text { sexual }\end{array}$ & Parcial o completamente heterosexual & 108 \\
& Parcial o completamente homosexual & 98 \\
$\begin{array}{l}\text { Estatus socio } \\
\text { económico } \\
\text { percibido }\end{array}$ & Pobre a clase media & 28 \\
& Clase media & 120 \\
\hline
\end{tabular}

\section{Resultados}

\section{Análisis factorial exploratorio de la escala de esencialismo}

Para evaluar la estructura de las creencias esencialistas en la población colombiana se realizó un análisis factorial que explicó el $83.18 \%$ de la varianza. Los ítems se cargaron en 2 factores que explicaban el $54.15 \%$ de la varianza. El primer factor, al que se denominará Fundamentalidad, que reúne las creencias en las características necesarias y definitorias, el potencial inductivo y la discreción de la categoría, pero también la creencia en la determinación temprana de la orientación sexual, explicó la mayor parte 39.5\% de la varianza (tabla 2). El segundo factor, Inmutabilidad reúne los ítems de determinismo biológico e inmutabilidad. La correlación entre los dos factores es de $.32 p<.000$.

Tabla 2

Análisis factorial de la escala de creencias esencialistas

\begin{tabular}{lcc}
\hline \multicolumn{1}{c}{ Escala } & Factor \\
\hline \multicolumn{1}{c}{$\begin{array}{c}\text { Fundamen- } \\
\text { talidad }\end{array}$} & $\begin{array}{c}\text { Inmutabi- } \\
\text { lidad }\end{array}$ \\
\hline $\begin{array}{l}\text { La homosexualidad es causada } \\
\text { por factores biológicos como } \\
\text { genes y hormonas }\end{array}$ & .362 & $\mathbf{. 7 5 2}$ \\
$\begin{array}{l}\text { Las personas no pueden cam- } \\
\text { biar su orientación sexual }\end{array}$ & .187 & $\mathbf{. 8 0 0}$ \\
$\begin{array}{l}\text { El que las personas sean ho- } \\
\text { mosexuales o heterosexuales }\end{array}$ & & \\
se determina en la infancia \\
temprana
\end{tabular}

Nota: las cargas factoriales superiores a 4 aparecen en negrita. 
Uno de los ítems no obtuvo una carga factorial superior a .4 en ningún factor, por lo que fue eliminado de la escala. Con los ítems restantes se elaboró la escala de Creencias Esencialistas sobre la Orientación Sexual, la cual obtuvo un buen índice de correlación interna $(\alpha=.73)$.

\section{Análisis de Varianza}

Al inicio del análisis se realizó una ANOVA, al tomar como variables independientes el sexo de los participantes y las variables independientes del modelo (percepción de estabilidad o cambio e identificación con el grupo normativo o no normativo). En este análisis no se encontraron resultados significativos para la variable sexo, ni en interacción con otras variables, por lo que fue excluida de análisis subsecuentes. El Análisis de Varianza incluyó entonces como variables independientes la manipulación de la percepción de cambio social (cambio, estabilidad, grupo control) y el estatus del grupo (orientación sexual normativa o no normativa). Como variable dependiente se introdujo la Escala de Creencias Esencialistas sobre la Orientación sexual. El efecto de la manipulación sobre las tres condiciones experimentales fue significativo $(F=2,205)=5.88, p<.01 \mathrm{ETA}$ $=0.56$, como también el efecto del estatus del grupo (normativo o no normativo), $(F=1,205)=$ 8.63, $p<.01, \mathrm{ETA}=0.41$. Tanto las personas en la condición de expectativa de cambio $(M=4.1, D E$ $=1.75, p<.01, \mathrm{ETA}=0.33)$ como las personas en la condición de estabilidad $(M=3.73, D E=1.39$, $p<.001, \mathrm{ETA}=0.70)$ reportaron menores niveles de pensamiento esencialista que las personas en la condición de control $(M=4.9, D E=2.02)$. Por otra parte, el grupo heterosexual respaldó creencias esencialistas de manera significativamente mayor que el grupo homosexual $(M=4.9, D E=1.7$ y 4.23 , $D E, 2.0$ respectivamente, $p<.01, \mathrm{ETA}=0.41)$. La interacción entre ambas variables, sin embargo, no fue significativa.

\section{Esencialismo y prejuicio heterosexual}

Para evaluar el papel de diferentes creencias esencialistas y la percepción de estabilidad o cambio en la predicción del prejuicio heterosexista, se corrió una serie de regresiones, incluyendo como factores predictores en primer lugar aspectos sociodemográficos (edad, género, estatus socioeconómico) seguidas de dos sub-escalas de la escala de esencialismo: inmutabilidad y fundamentalidad. La escala de Inmutabilidad incluye la creencia en la determinación biológica y la inmutabilidad $(\alpha=.5)$. La escala de Fundamentalidad incluye las demás creencias, conforme a los resultados del análisis factorial $(\alpha=.73)$.

Adicionalmente, teniendo en cuenta los resultados de la sección anterior, según los cuales no hay interacciones significativas entre las condiciones experimentales de cambio y estabilidad y la orientación sexual, se crearon dos variables dicótomas de control que dan cuenta de la participación de las personas en las condiciones experimentales $(1=\mathrm{el}$ participante recibió alguna de las manipulaciones experimentales de las condiciones de estabilidad y de cambio, $0=$ el participante no recibió manipulación experimental), se crearon términos de interacción para evaluar el posible papel moderador de la percepción de cambio en la relación entre esencialismo y prejuicio y se corrieron simultáneamente regresiones separadas para el grupo normativo y no normativo.

Las creencias esencialistas parecen cumplir un papel más significativo en la población heterosexual que en la homosexual, en la predicción del prejuicio hacia los hombres y las mujeres homosexuales. En el caso del prejuicio en contra de mujeres y hombres homosexuales, la edad y la creencia en la fundamentalidad de la orientación sexual predicen mayores niveles de prejuicio (tablas 3 y 4) entre heterosexuales. Entre homosexuales, en cambio, ninguna variable de las introducidas en el modelo se relaciona significativamente con el prejuicio. 
Tabla 3

Prejuicio en contra de los hombres homosexuales

\begin{tabular}{|c|c|c|c|c|c|c|c|c|}
\hline & \multicolumn{3}{|c|}{ Heterosexuales } & \multicolumn{5}{|c|}{ Homosexuales } \\
\hline & $B$ & Std. Error & Beta & Sig. & $B$ & Std. Error & Beta & Sig. \\
\hline (Constant) & .790 & 1.202 & & & 1.705 & .837 & & * \\
\hline Género & -.020 & .499 & -.004 & & .098 & .273 & .037 & \\
\hline SES & .000 & .000 & -.032 & & -.074 & .166 & -.046 & \\
\hline Edad & .036 & .017 & .217 & $*$ & .004 & .015 & .027 & \\
\hline Inmutabilidad & -.043 & .121 & -.049 & & .071 & .087 & .130 & \\
\hline Fundamentalidad & .378 & .181 & .304 & $*$ & .002 & .096 & .004 & \\
\hline Estabilidad & 1.909 & 1.987 & .336 & & .141 & .978 & .043 & \\
\hline Cambio & 3.018 & 2.025 & .520 & & -.749 & .863 & -.237 & \\
\hline Int1 & -.059 & .309 & -.060 & & .419 & .249 & .386 & \\
\hline Int2 & -.062 & .250 & -.045 & & -.272 & .179 & -.412 & \\
\hline Int3 & -.067 & .397 & -.067 & & .177 & .220 & .243 & \\
\hline Int2 & -.206 & .291 & -.141 & & -.008 & .163 & -.012 & \\
\hline $\mathrm{R}$ & .562 & & & & .281 & & & \\
\hline Rcuadrado & .316 & & & & .079 & & & \\
\hline Rajustada & .213 & & & & -.027 & & & \\
\hline
\end{tabular}

Nota: $p<.05^{*} ; p<.01 * * ; p<.001 * * *$. Int1 = Extabilidad x Fundamentalidad; Int2 = Estabilidad $\mathrm{x}$ Inmutabilidad; Int3=Cambio $\mathrm{x}$ Fundamentalidad; Int 4 = Cambio $x$ Inmutabilidad.

Tabla 4

Prejuicio en contra de las mujeres homosexuales

\begin{tabular}{|c|c|c|c|c|c|c|c|c|}
\hline & \multicolumn{3}{|c|}{ Heterosexuales } & \multicolumn{5}{|c|}{ Homosexuales } \\
\hline & $B$ & Std. Error & Beta & Sig. & $B$ & Std. Error & Beta & Sig. \\
\hline (Constant) & -.500 & 1.198 & & & .991 & .854 & & \\
\hline Género & .170 & .497 & .032 & & .070 & .277 & .024 & \\
\hline SES & .000 & .000 & -.002 & & -.079 & .170 & -.045 & \\
\hline Edad & .058 & .017 & .323 & $* *$ & .015 & .015 & .092 & \\
\hline Inmutabilidad & -.197 & .121 & -.208 & & -.026 & .089 & -.045 & \\
\hline Fundamentalidad & .518 & .181 & .384 & $* *$ & .181 & .099 & .269 & \\
\hline Estabilidad & 1.765 & 1.980 & .285 & & -.160 & .954 & -.046 & \\
\hline Cambio & 2.485 & 2.018 & .394 & & -1.049 & .883 & -.304 & \\
\hline Int1 & -.079 & .308 & -.073 & & .479 & .250 & .423 & \\
\hline Int2 & -.029 & .249 & -.019 & & -.151 & .178 & -.215 & \\
\hline Int3 & -.018 & .395 & -.016 & & .138 & .225 & .173 & \\
\hline
\end{tabular}




\begin{tabular}{|c|c|c|c|c|c|c|c|c|}
\hline & \multicolumn{3}{|c|}{ Heterosexuales } & \multicolumn{5}{|c|}{ Homosexuales } \\
\hline & $B$ & Std. Error & Beta & Sig. & $B$ & Std. Error & Beta & Sig. \\
\hline Int2 & -.266 & .290 & -.167 & & .153 & .167 & .221 & \\
\hline $\mathrm{R}$ & .653 & & & & .424 & & & \\
\hline Rcuadrado & .426 & & & & .180 & & & \\
\hline Rajustada & .340 & & & & .087 & & & \\
\hline
\end{tabular}

Nota: $p<.05^{*} ; p<.01 * * ; p<.001 * * *$. Int $1=$ Extabilidad $\mathrm{x}$ Fundamentalidad; Int2=Estabilidad $\mathrm{x}$ Inmutabilidad; Int3=Cambio $\mathrm{x}$ Fundamentalidad; Int $4=$ Cambio $x$ Inmutabilidad.

Esencialismo y actitudes hacia el matrimonio igualitario y la adopción por parejas homosexuales

Para evaluar el papel de diferentes creencias esencialistas en la las actitudes hacia el matrimonio igualitario y la adopción por parejas homosexuales, en población normativa (heterosexual) y no normativa (homosexual), se corrió una serie de regresiones incluyendo como factores predictores, en primer lugar, aspectos sociodemográficos (edad, género, estatus socioeconómico) las creencias esencialistas en inmutabilidad, la determinación temprana y el prejuicio en contra de hombres y mujeres homosexuales. Nuevamente, se incluyeron las dos variables dicótomas de control que dan cuenta de la participación de las personas en las condiciones experimentales y las interacciones entre esencialismo y las condiciones de cambio o estabilidad.

En ambos casos (matrimonio y adopción) se encontró que en la población heterosexual la creencia en la determinación temprana de la orientación sexual impacta de manera negativa y significativa las actitudes tanto hacia el matrimonio igualitario como hacia la adopción por parejas homosexuales. Así mismo, la expectativa de cambio inminente en el sistema y la edad.

Tabla 5

Actitudes hacia el matrimonio igualitario

\begin{tabular}{|c|c|c|c|c|c|c|c|c|}
\hline & \multicolumn{4}{|c|}{ Heterosexuales } & \multicolumn{4}{|c|}{ Homosexuales } \\
\hline & $B$ & Std. Error & Beta & Sig. & $B$ & Std. Error & Beta & Sig. \\
\hline (Constant) & 12.831 & 1.527 & & $* * *$ & 8.384 & 1.400 & & $* * *$ \\
\hline Género & -1.449 & .634 & -.210 & $*$ & -.636 & .455 & -.137 & \\
\hline SES & .000 & .000 & -.027 & & .317 & .278 & .112 & \\
\hline Edad & -.070 & .022 & -.300 & $* *$ & -.008 & .025 & -.032 & \\
\hline Inmutabilidad & .040 & .154 & .032 & & .063 & .145 & .066 & \\
\hline Fundamentalidad & -.719 & .230 & -.406 & $* *$ & -.156 & .161 & -.145 & \\
\hline Estabilidad & -3.057 & 2.524 & -.377 & & .008 & 1.561 & .001 & \\
\hline Cambio & -6.764 & 2.572 & -.818 & $* *$ & 1.171 & 1.460 & .209 & \\
\hline Int 1 & .031 & .392 & .022 & & -.760 & .408 & -.421 & \\
\hline Int2 & .487 & .317 & .245 & & .359 & .292 & .321 & \\
\hline
\end{tabular}




\begin{tabular}{|c|c|c|c|c|c|c|c|c|}
\hline & \multicolumn{4}{|c|}{ Heterosexuales } & \multicolumn{4}{|c|}{ Homosexuales } \\
\hline & $B$ & Std. Error & Beta & Sig. & $B$ & Std. Error & Beta & Sig. \\
\hline Int3 & .718 & .504 & .504 & & .233 & .384 & .176 & \\
\hline Int2 & .220 & .370 & .105 & & -.461 & .274 & -.409 & \\
\hline $\mathrm{R}$ & .676 & & & & .378 & & & \\
\hline Rcuadrado & .457 & & & & .143 & & & \\
\hline Rajustada & .375 & & & & .045 & & & \\
\hline
\end{tabular}

Nota: $p<.05 * ; p<.01 * * ; p<.001 * * *$. Int $1=$ Estabilidad $\mathrm{x}$ Fundamentalidad; Int2=Estabilidad $\mathrm{x}$ Inmutabilidad; Int3=Cambio $\mathrm{x}$ Fundamentalidad; Int $4=$ Cambio $x$ Inmutabilidad.

Tabla 6

Actitudes hacia la adopción por pareja homosexuales

\begin{tabular}{|c|c|c|c|c|c|c|c|c|}
\hline & \multicolumn{3}{|c|}{ Heterosexuales } & \multicolumn{5}{|c|}{ Homosexuales } \\
\hline & $B$ & Std. Error & Beta & Sig. & $B$ & Std. Error & Beta & Sig. \\
\hline (Constant) & 11.185 & 1.506 & & $* * *$ & 6.706 & 1.569 & & $* * *$ \\
\hline Género & -1.261 & .625 & -.192 & $*$ & .248 & .509 & .048 & \\
\hline SES & .000 & .000 & -.035 & & .423 & .312 & .134 & \\
\hline Edad & -.069 & .021 & -.311 & $* *$ & -.009 & .028 & -.030 & \\
\hline Inmutabilidad & -.024 & .152 & -.020 & & -.034 & .163 & -.032 & \\
\hline Fundamentalidad & -.497 & .227 & -.296 & $* *$ & -.138 & .181 & -.115 & \\
\hline Estabilidad & -1.866 & 2.491 & -.242 & & -.631 & 1.754 & -.101 & \\
\hline Cambio & -6.140 & 2.538 & -.781 & $* *$ & 2.346 & 1.624 & .381 & \\
\hline Int1 & -.185 & .387 & -.138 & & -.734 & .459 & -.363 & \\
\hline Int2 & .560 & .313 & .297 & & .484 & .328 & .387 & \\
\hline Int3 & .661 & .497 & .489 & & -.359 & .414 & -.253 & \\
\hline Int2 & .075 & .365 & .038 & & -.128 & .306 & -.103 & \\
\hline $\mathrm{R}$ & .644 & & & & .358 & & & \\
\hline Rcuadrado & .414 & & & & .128 & & & \\
\hline Rajustada & .326 & & & & .029 & & & \\
\hline
\end{tabular}

Nota: $p<.05^{*} ; p<.01 * * ; p<.001 * * *$. Int $1=$ Estabilidad $\mathrm{x}$ Fundamentalidad; Int2=Estabilidad $\mathrm{x}$ Inmutabilidad; Int3=Cambio $\mathrm{x}$ Fundamentalidad; Int $4=$ Cambio $x$ Inmutabilidad.

$\mathrm{Al}$ incluir en el modelo de regresión las variables de prejuicio, se encontró que estas aportan también de manera significativa en la predicción de las actitudes negativas hacia el matrimonio y la adopción. Adicionalmente, los niveles de significación de las creencias esencialistas disminuyen, de manera que solo el efecto de la creencia en la determinación temprana sobre las actitudes hacia el matrimonio sigue siendo significativo. Adicionalmente, llama la atención que tanto los coeficientes como los niveles de significación son mayores en el caso del prejuicio en contra de mujeres lesbianas, en relación con las actitudes hacia la adopción. Esto es cierto incluso en la población homosexual. 


\section{Discusión}

Esta investigación tuvo como propósito evaluar el efecto de la percepción de un cambio social inminente en el estatus de un grupo históricamente subordinado (población homosexual), sobre la tendencia a utilizar argumentos de tipo esencialista, dependiendo del estatus normativo o no normativo del evaluador. Para lograrlo, fue necesario también evaluar la estructura de las creencias esencialistas en una muestra de comunidad colombiana. La intuición que subyace a este propósito se origina en los hallazgos recientes sobre el esencialismo psicológico, los cuales sugieren que el pensamiento esencialista sobre la gente gay no siempre acompaña el prejuicio social y de hecho puede estructurarse de diferentes maneras en diferentes contextos, así como en grupos de diferente estatus. Adicionalmente, algunas investigaciones previas han reducido el concepto a las dimensiones biológicas de este, o bien limitaban sus muestras a población representativa de la mayoría, por lo que no era posible apreciar la variabilidad entre grupos de una misma sociedad.

En esta investigación se intenta dar cuenta de estas limitaciones, para lo cual se adaptó una escala de esencialismo que incluía los ítems tradicionalmente utilizados para medir creencias esencialistas teniendo como base las propuestas por Haslam et al. (2000, 2002), Haslam y Levy (2006) y Hegarty y Pratto (2006). Adicionalmente, se incluyó una muestra de población representativa de la mayoría (normativa), pero también una muestra representativa de un grupo históricamente subordinado (no normativo) y se contrastó el efecto de las creencias esencialistas sobre sus actitudes hacia la población homosexual y algunos temas de relevancia social como el matrimonio igualitario y el derecho a la adopción por parejas homosexuales.

En primer lugar, el análisis factorial exploratorio realizado con la totalidad de la muestra arrojó resultados comparables a los encontrados en otras sociedades: las creencias en la informati-

Tabla 7

Actitudes hacia la adopción

\begin{tabular}{|c|c|c|c|c|c|c|c|c|}
\hline & \multicolumn{3}{|c|}{ Heterosexuales } & \multicolumn{5}{|c|}{ Homosexuales } \\
\hline & $B$ & Std. Error & Beta & Sig. & $B$ & Std. Error & Beta & Sig. \\
\hline (Constant) & 11.707 & 1.184 & & $* * *$ & 9.280 & 1.082 & & $* * *$ \\
\hline Género & -1.061 & .523 & -.154 & $*$ & -.418 & .355 & -.089 & \\
\hline SES & .000 & .000 & -.018 & & .242 & .214 & .086 & \\
\hline Edad & -.038 & .020 & -.163 & $*$ & .015 & .020 & .057 & \\
\hline Inmutabilidad & .080 & .104 & .065 & & .022 & .087 & .024 & \\
\hline Fundamentalidad & -.334 & .151 & -.189 & $*$ & .026 & .102 & .024 & \\
\hline Prejuicio antigay & -.371 & .171 & -.260 & $*$ & -.087 & .230 & -.048 & \\
\hline Prejuicio antilesbianas & -.435 & .175 & -.332 & $*$ & -1.045 & .219 & -.620 & $* * *$ \\
\hline $\mathrm{R}$ & .762 & & & & .671 & & & \\
\hline Rcuadrado & .581 & & & & .450 & & & \\
\hline Rajustada & .542 & & & & .411 & & & \\
\hline
\end{tabular}

Nota: $p<.05^{*} ; p<.01^{* *} ; p<.001^{* * *}$. Int $1=$ Estabilidad $\mathrm{x}$ Fundamentalidad; Int2=Estabilidad $\mathrm{x}$ Inmutabilidad; Int3=Cambio $\mathrm{x}$ Fundamentalidad; Int4 = Cambio $\mathrm{x}$ Inmutabilidad. 
Tabla 8

Actitudes hacia la adopción

\begin{tabular}{|c|c|c|c|c|c|c|c|c|}
\hline & \multicolumn{3}{|c|}{ Heterosexuales } & \multicolumn{5}{|c|}{ Homosexuales } \\
\hline & $B$ & Std. Error & Beta & Sig. & $B$ & Std. Error & Beta & Sig. \\
\hline (Constant) & 10.457 & 1.205 & & $* * *$ & 7.977 & 1.337 & & $* * *$ \\
\hline Género & -.838 & .532 & -.128 & & .424 & .436 & .081 & \\
\hline SES & .000 & .000 & -.022 & & .338 & .264 & .107 & \\
\hline Edad & -.039 & .020 & -.174 & $*$ & .004 & .024 & .012 & \\
\hline Inmutabilidad & .047 & .106 & .039 & & .010 & .108 & .009 & \\
\hline Fundamentalidad & -.246 & .153 & -.146 & & -.078 & .126 & -.065 & \\
\hline Prejuicio antigay & -.429 & .174 & -.317 & $*$ & -.422 & .283 & -.216 & \\
\hline Prejuicio antilesbianas & -.325 & .178 & -.261 & & -.606 & .269 & -.340 & * \\
\hline $\mathrm{R}$ & .720 & & & & .567 & & & \\
\hline Rcuadrado & .519 & & & & .321 & & & \\
\hline Rajustada & .475 & & & & .273 & & & \\
\hline
\end{tabular}

Nota: $p<.05^{*} ; p<.01 * * ; p<.001 * * *$. Int $1=$ Estabilidad $\mathrm{x}$ Fundamentalidad; Int2=Estabilidad $\mathrm{x}$ Inmutabilidad; Int3=Cambio $\mathrm{x}$ Fundamentalidad; Int $4=$ Cambio $x$ Inmutabilidad.

vidad, fundamentalidad y el potencial inductivo de la categoría cargan en un mismo factor, mientras que las creencias en el determinismo biológico y la inmutabilidad cargan en un segundo factor, ambos factores correlacionados positivamente. En este sentido, se encontró que el pensamiento esencialista sobre la orientación sexual, definido en los términos en que tradicionalmente se ha hecho en la literatura, está vigente de manera significativa en la sociedad colombiana. Las dimensiones subyacentes se acercan suficientemente a las encontradas en otras investigaciones, aunque aparece una diferencia importante también. En este caso, la creencia en la determinación temprana de la orientación sexual carga sobre el factor de Fundamentalidad y no sobre el de Inmutabilidad. Esta diferencia sugiere que algunas creencias esencialistas en este contexto se ordenan alrededor de la Creencia en la Determinación Temprana o Social, conceptos propuestos por Hegarty y Pratto, 2001 y por Rangel y Keller (2009), respectivamente, y no tanto alrededor de la Creencia en la Determinación biológica como en otras sociedades. Esta hipótesis requiere ser evaluada a la luz de la evidencia empírica en un estudio con mayor alcance. Por otra parte, es posible que las creencias esencialistas no se distribuyan de manera uniforme en toda la población o no tengan la misma relevancia e implicaciones para todos los grupos que conforman la sociedad colombiana.

La teoría de la justificación del sistema plantea que las ideologías justificadoras del sistema cumplen una función de protección del individuo, en tanto que le permiten mantener la noción de que el mundo que le rodea es predecible y seguro. En consecuencia, se intuye que si las creencias esencialistas cumplen un papel justificador, la percepción de estabilidad o inminencia en el cambio social debería afectar la tendencia a respaldar creencias esencialistas entre participantes de grupos socialmente normativos y no normativos. En particular, se esperaba encontrar mayor respaldo esencialista entre quienes percibían que el cambio era inminente. En contraste, se encontró que, comparado con las personas que no recibieron manipulación experimental, el reporte de creencias esencialistas en am- 
bos grupos experimentales fue significativamente menor, lo cual es explicado por una disminución en el reporte de estas creencias entre los participantes homosexuales comparados con el grupo control, $y$ no por un aumento en el reporte de creencias esencialistas de los participantes heterosexuales, el cual se mantuvo estable. Este sorprendente hallazgo cualificado por los análisis de regresión realizados merece una mayor exploración.

En el grupo normativo (heterosexuales) los análisis de regresión sugieren que las creencias en la determinación biológica y la inmutabilidad no están asociadas al prejuicio anti-gay, por otra parte, las creencias en la informatividad, fundamentalidad y el potencial inductivo de la categoría, junto con la creencia en la determinación temprana, reunidas en el factor Fundamentalidad correlacionan de manera estable y significativa con el prejuicio en contra de las personas homosexuales en general, y predicen actitudes negativas hacia el matrimonio igualitario y hacia la adopción por parejas homosexuales. Estos hallazgos son consistentes con los de Hegaty y Pratto (2001), que sugieren que diferentes creencias sobre la orientación sexual, todas ellas de carácter esencialista, pueden tener diferentes implicaciones en la evaluación de determinados grupos sociales.

Hegarty y Pratto encontraron, sin embargo, que la creencia en la inmutabilidad de la orientación sexual correlacionaba con actitudes más favorables hacia los homosexuales. Los hallazgos aquí reportados no respaldan la noción de una correlación pero sí el que la creencia en la inmutabilidad no conlleva al prejuicio. Por otra parte, la creencia en la fundamentalidad, discreción, potencial inductivo (igual que en los estudios de Hegarty y Pratto), pero también la creencia en la determinación temprana de la categoría, predicen el prejuicio hacia las personas homosexuales de ambos géneros. En este sentido, se requiere mayor investigación para explorar la estructura del sistema de creencias vigente en la sociedad colombiana, la cual a juzgar por los hallazgos de este estudio puede señalar diferencias culturales importantes. Más estudios de este tipo deberían realizarse en sociedades con trayectorias culturales similares para evaluar esta hipótesis.

Continuando con la discusión sobre la variabilidad del pensamiento esencialista, los hallazgos de esta investigación contribuyen al argumento levantado en investigaciones recientes que sugieren que el pensamiento esencialista varía en su estructura y en sus implicaciones dependiendo del estatus relativo de los grupos. Cuando se incorpora la participación de personas que pertenecen a grupos normativos y no normativos, es posible apreciar con claridad las diferencias en el uso y las implicaciones del pensamiento esencialista, lo cual sugiere que el esencialismo cumple en efecto una función de justificación del sistema. Este hallazgo fortalece los de estudios previos como el de Jayaratne, et al., (2006, 2009), Mahalingam y Rodríguez, (2003), Morton et al., (2009a y 2009b) Verkuyten (2003), Mahalingam y otros. La evidencia aquí presentada sugiere que el pensamiento esencialista cumple un papel relevante como sistema de creencias que contribuye a organizar el mundo social. No obstante, estas creencias no se distribuyen de manera uniforme en toda la población, ni tienen las mismas implicaciones para grupos normativos y no normativos. En este caso particular, las creencias esencialistas en la fundamentalidad y en la determinación temprana de la orientación sexual parecen tener un efecto negativo sobre las actitudes de los participantes pertenecientes al grupo normativo, pero no tienen el mismo efecto sobre los participantes del grupo no normativo.

Finalmente, otro aspecto que llama la atención de los resultados aquí presentados y que merece mayor exploración tiene que ver con las implicaciones del pensamiento esencialista para personas homosexuales de diferente género. De manera estable, en los hallazgos de esta investigación se encontró que el prejuicio en contra de mujeres homosexuales predice con mayor fuerza las actitudes negativas hacia el matrimonio y la adopción. Se requieren más estudios que diferencien las implicaciones del prejuicio para diferentes subgrupos de la comuni- 
dad gay para evaluar la validez de este hallazgo, así como la posibilidad de que, como lo han señalado otros autores en el pasado, diferentes dimensiones de identificación pero también de subordinación se activen simultáneamente, como en este caso, la orientación sexual y el género, posiblemente empeorando las implicaciones para este grupo.

Por último, es importante señalar, entre las limitaciones de este estudio, el tamaño de los grupos experimentales, comparado con el del grupo control. Este obedece a un cambio en las condiciones del entorno, que consistió en que a mediados de 2013 lo que hasta ese momento era una posibilidad de cambio social se materializó cuando el matrimonio gay se hizo posible debido a un fallo de la Corte Constitucional en junio de 2011 en ese sentido. Teniendo en cuenta este cambio, se detuvo la recolección de información de los grupos experimentales dado que, frente a la realidad, la efectividad de las manipulaciones experimentales quedaba seriamente cuestionada.

\section{Referencias}

Arseneau, J., Grzanka, P., Miles, J., \& Fassinger, R. (2013). Development and Initial Validation of the Sexual Orientation Beliefs Scale (SOBS). Journal of Counseling Psychology, 60(3), 407-20.

Blasi, G., \& Jost, J. (2006). System Justification Theory and Research: Implications for Law, Legal Advocacy, and Social Justice. California Law Review, 94(4), 1119-1168.

Dar-Nimrod, I., \& Heine, S. (2011). Genetic essentialism: on the deceptive determinism of DNA. Psychological bulletin, 137(5), 800-18.

Estrada, A., Acuña, M., Camino L., \& Traverso, M. (2007). ¿Se nace o se hace? Repertorios interpretativos sobre la homosexualidad en Bogotá. Revista de Estudios Sociales, 28, 56-71.

Estrada, C., Oyarzún, M., \& Yzerbyt, V. (2007). Teorías Implícitas y Esencialismo Psicológico: Herramientas Conceptuales Para el Estudio de las Relaciones Entre y Dentro de los Grupos. Psykhe, 16(1), 111-121.

Gelman, S. A. (2003). The essential child: Origins of essentialism in everyday thought. New York: Oxford University Press.

Haider-Markel, D., \& Joslyn, M. (2008). Beliefs about the Origins of homosexuality and support for Gay rights: An empirical test of attribution theory. Public Opinion Quarterly, 72(2), 291310.

Haslam, N., Rothschild, L., \& Ernst, D. (2000). Essentialist beliefs about social categories. The British journal of social psychology, 39(1), 113-127.

Haslam, N., Rothschild, L., \& Ernst, D. (2002). Are essentialist beliefs associated with prejudice? The British journal of social psychology, 41(1), $87-100$.

Haslam, N., \& Levy, S. (2006). Essentialist beliefs about homosexuality: structure and implications for prejudice. Personality \& Social Psychology Bulletin, 32(4), 471-485.

Hegarty, P., \& Golden, A. (2008). Attributional Beliefs About the Controllability of Stigmatized Traits: Antecedents or Justifications of Prejudice? Journal of Applied Social Psychology, 38(4), 1023-1044.

Hegarty, P., \& Pratto, F. (2006). Sexual Orientation Beliefs: Their Relationship to Anti-Gay Attitudes and Biological Determinist Arguments. Journal of Homosexuality, 41(1), 121-35.

Heider, F. (1958). The psychology of interpersonal relations. New York: John Wiley \& Sons.

Herek, G. (1998). Attitudes Toward Lesbians and Gay Men Scale. En C. Davis, W. Yaber, R. Bauserman, G. Schreer \& S. Davis (Eds.), Handbook of Sexuality-Related Measures (pp. 392-395). Londres: Sage.

Hirschfeld, L. (2002). ¿La adquisición de categorías sociales se basa en una competencia de dominio específico o en la transferencia de conocimientos? En L. Hirschfeld \& S. Gelman (Eds.), Cartografía de la mente: la especificidad de 
dominio en la cognición y la cultura (pp. 285328). Barcelona: Gedisa.

Jayaratne, T., Gelman, S., Felbaum, M., Sheldon, J. P., Petty, E. M., \& Kardia, S. L. R. (2009) The perennial debate: Nature, nurture or choice? Black and White Americans' explanations for individual differences. Review of General Psycholgy, 13, 24-33.

Jayaratne, T., Ybarra, O., Sheldon, J., Brown, T., Feldbaum, M., Pfeffer, C., \& Petty, E. (2006). White Americans' Genetic Lay Theories of Race Differences and Sexual Orientation: Their Relationship with Prejudice toward Blacks, and Gay Men and Lesbians. Group Processes \& Intergroup Relations, 9(1), 77-94.

Jost, J., Banaji, M., \& Nosek, B. (2004). A Decade of System Justification Theory: Accumulated Evidence of Conscious and Unconscious Bolstering of the Status Quo. Political Psychology, 25, 881-919.

Jost, J., Pelham, B., Sheldon, O., \& Sullivan, B. (2003). Social Inequality and the Reduction of Ideological Dissonance on Behalf of the System: Evidence of Enhanced System Justification Among the Disadvantaged. European Journal of Social Psychology, 33, 13-36.

Keller, J. (2005). In Genes We Trust: The Biological Component of Psychological Essentialism and Its Relationship to Mechanisms of Motivated Social Cognition. Journal of personality and social psychology, 88(4), 686-702.

Mahalingam, R., \& Rodríguez, J. (2003). Essentialism, Power and Cultural Psychology of Gender. Journal of Cognition and Culture, 3(2), 157-174.

Mahalingam, R., \& Rodríguez, J. (2006). Culture, brain transplants and implicit theories of identity. Journal of Cognition and Culture, 6(3-4), 453-462.

Medin, D., \& Ortony, A. (1989). Psychological Essentialism. En S. Vosniadou \& A. Ortony (Eds.), Similarity and Analogical Reasoning (pp. 179195). Nueva York: Cambridge University Press.
Morton, T., Hornsey, M. J., \& Postmes, T. (2009a). Shifting ground: The variable use of essentialism in contexts of inclusion and exclusion. The British Journal of Social psychology, 48(1), 35-59.

Morton, T., Postmes, T., Haslam, S., \& Hornsey, M. (2009b). Theorizing Gender in the Face of Social Change: Is There Anything Essential About Essentialism? Journal of Personality and Social Psychology, 96(3), 653-664.

Pereira, M., Alvaro J., \& Schweiger I. (2010). Essentialism and the Expression of Social Stereotypes: a Comparative Study of Spain, Brazil and England. The Spanish Journal of Psychology, 13(2), 808-17.

Ramírez, L., Vega, A., \& Moreno, P. (2013, Julio) Creencias esencialistas, prejuicio y estigmatización de la orientación sexual gay. Presentación oral realizada en el Congreso Interamericano de Psicología, Brasilia, Distrito Federal, Brasil. Recuperado de http://www.sip2013.org/pdf/ ANAIS_CIP_geral(draft).pdf

Rangel, U., \& Keller, J. (2011). Essentialism Goes Social: Belief in Social Determinism as a Component of Psychological Essentialism. Journal of Personality and Social Psychology, 100(6), 1056-1078.

Tsukamoto, S., Enright, J., \& Karasawa, M. (2013). Psychological Essentialism and Nationalism as Determinants of Interethnic Bias. The Journal of Social Psychology, 155(5), 515-519.

Verkuyten, M. (2003). Discourses about ethnic group (de-)essentialism: Oppressive and progressive aspects. The British Journal of Social Psychology / The British Psychological Society, 42(Pt 3), 371-391.

Yzerbyt, V., Rocher, S., \& Schadron, G. (1997). Stereotypes as Explanations: A Subjective Essentialistic View of Group Perception. En R. Spears, P. Oakes, N. Ellemers, \& S. Haslam (Eds.), The Social Psychology of Stereotyping and Group Life (pp. 20-50). Oxford: Blackwell 


\section{Received: April 3, 2014 \\ ficcepted: September 17, 2014}

\section{ANEXO \\ Ejemplo de manipulación experimental}

\section{Versión Cambio Inminente. Homosexuales: cada día más cerca de alcanzar la igualdad en Colombia}

En muchos países del mundo las relaciones entre parejas gay son aceptadas por la sociedad. En Colombia la homosexualidad ya no es un tabú y es más aceptada por muchas personas.

La homosexualidad es un tema polémico que genera división. Actualmente, nuestro país debate si se debe aprobar el matrimonio de parejas de un mismo sexo. A raíz de la importancia de este tema para la coyuntura nacional, realizamos una revisión de la situación de la homosexualidad en Colombia y en el mundo.

Según un informe realizado por la Asociación Mundial de Lesbianas y Gays (WLGA, por sus siglas en inglés) la situación de los homosexuales ha mejorado en los últimos años. En 113 países del mundo se considera que la homosexualidad es legal. En once países se permite el matrimonio entre dos personas del mismo sexo, y en 14 se admite la adopción conjunta de una pareja de igual género. En América Latina dos países, Argentina y México, tienen legalizados el matrimonio gay y la adopción.

En Colombia, la legislación favorece en muchos aspectos a las personas homosexuales. La Corte Constitucional ha establecido que una persona no puede ser discriminada por su orientación sexual. Gays y lesbianas no pueden ser despedidos de sus cargos por este motivo. Desde el 2007 la Corte reconoce derechos en salud y pensión a las parejas del mismo sexo. Esto significa que las parejas homosexuales pueden heredar, beneficiarse mutuamente para recibir seguridad social y constituir uniones civiles ante una notaría. Esta legislación se cumple cada día más y las parejas del mismo sexo ya no tienen que recurrir a acciones legales para hacer valer sus derechos.

El 26 de junio de 2011 la Corte reconoció el derecho de los homosexuales a conformar una familia y a establecer una unión formal con los mismos derechos que los de las uniones heterosexuales. En su sentencia, la Corte le dio plazo al Congreso hasta el 20 de junio de 2013 para regular este tema. Actualmente, cursa en el Congreso un Proyecto de Ley para legislar al respecto y todo apunta a que esta iniciativa será aprobada.

La legislación colombiana se caracteriza, a nivel mundial, por ser una de las más favorables hacia la comunidad LGBTI. Adicionalmente, las normas culturales y sociales existentes en el país cada día son más tolerantes con respecto a la homosexualidad. Han disminuido las manifestaciones de prejuicio hacia mujeres y hombres de la comunidad Gay. Por ejemplo, las marchas del Orgullo Gay que desfilan por las calles son cada vez más respetadas por la mayoría de ciudadanos. Hoy en día la homosexualidad es aceptada por la sociedad como una orientación sexual válida.

La incorporación de la población homosexual a la normalidad cotidiana está cerca de alcanzarse. Cada día, más ciudadanos se atreven a romper tabúes. Muchos homosexuales han podido contarle a sus 
amigos, padres, hermanos, e incluso hijos, acerca de su orientación sexual encontrando en ellos apoyo y aprobación. Varias organizaciones y colectivos, con participación de reconocidas figuras homosexuales, defienden la causa de los derechos de las parejas del mismo sexo. Se sabe de bares y barrios en Bogotá, Barranquilla y Cali donde las parejas homosexuales son bienvenidas.

El logro más significativo que están alcanzado los homosexuales es convivir con el resto de la comunidad sin discriminaciones ni señalamientos. La clandestinidad ya no es una condición a la que hombres y mujeres homosexuales se ven obligados porque ahora cuentan con la protección de su libertad y sus derechos esenciales. 